\title{
Existence of Solution to Generalized Multivalued Vector Variational-like Inequalities
}

\author{
$\mathrm{Di} \mathrm{Wu}{ }^{1}$ \\ ${ }^{1}$ Department of Information Engineering, Chaoyang \\ Teachers Colleg, Chaoyang, Liaoning 122000, China \\ Email: wd8020@163.com.
}

\author{
Lu Shen $^{2}$, Ya-li ZhAO ${ }^{2} *$ \\ ${ }^{2}$ College of Mathematics and Physics, Bohai University, \\ Jinzhon , Liaoning 121013, China \\ *Corresponding author Email: yalizhao2000@163.com.
}

\begin{abstract}
In this paper, we consider a generalized multivalued vector variational-like inequality and obtain some existence results. some special cases are also discussed.

Keywords-Generalized multivalued vector variational-like inequality; Existence result; Affine mapping; Lower semicontinuity

\section{INTRODUCTION AND PRELIMINARIES}

Let $Y$ be a Banach space with a convex cone $P$ such that int $P \neq \phi$ and $P \neq Y$, where int denotes the interior. We use the following vector ordering: for any $x, y \in Y, y<x$ if and only if $y-x \in-\operatorname{int} P ; y \nless x$ if and only if $y-x \notin-$ int $P$. Let $X$ be a nonempty subset of a Banach space $E$ and $Y$ a Banach space with a convex cone $P$ such that int $P \neq \phi$ and $P \neq Y$. Let $N: L(E, Y) \times L(E, Y) \times L(E, Y) \rightarrow L(E, Y)$ be a singlevalued mapping, $M, S, T: X \rightarrow 2^{L(E, Y)}$ be three set-valued mappings, where $L(E, Y)$ is the space of all linear continuous mappings from $E$ into $Y$, and $\langle l, x\rangle$ denotes the value of $l$ at $X, \eta: X \times X \rightarrow Y, H: X \times X \rightarrow Y$ be two bimappings. We consider the following two generalized multivalued vector variational-like inequality, for short, denoted by GMVVLI-1 and GMVVLI-2, respectively.
\end{abstract}

GMVVLI-1: Find $y \in X$, such that for any

$$
\begin{gathered}
u \in M(y), v \in S(y), w \in T(y) \\
\langle N(u, v, w), \eta(x, y)\rangle+H(x, y) \nless 0, \forall x \in X .
\end{gathered}
$$

satisfying

GMVVLI-2: Find $y \in X$,

such that $\exists u \in M(y), v \in S(y), w \in T(y)$ satisfying $\langle N(u, v, w), \eta(x, y)\rangle+H(x, y) \nless 0, \forall x \in X$.

It is easy to see that each solution of GMVVLI-1 is that of GMVVLI-2, but the converse maybe not true in general. The aim of this paper is to derive the existence results of GMVVLI-1. It is well known that GMVVLI-1 and GMVVLI-

This work is supported by the National Natural Science Foundation of China (11371070).
2 are extensions of the vector variational inequality, which is a generalized form of a variational inequality, having applications in different areas of optimization, optimal control, operations research, economics equilibrium and free boundary value problems, see, for instance, [1-3] and the references therein. Now we give some definitions and lemma needed for the proof of the existence results.

Definition 1.1 [4] Let $X$ be a subset of a topological space $E$. Then a set-valued mapping $F: X \rightarrow 2^{E}$ is called the KKM mapping if for each finite subset $\left\{x_{1}, x_{2}, \cdots, x_{n}\right\}$ of $X \quad$, co $\left\{x_{1}, x_{2}, \cdots, x_{n}\right\} \subset \bigcup_{i=1}^{n} F\left(x_{i}\right)$, where co $\left\{x_{1}, x_{2}, \cdots, x_{n}\right\}$ is the convex hull of $\left\{x_{1}, x_{2}, \cdots, x_{n}\right\}$.

Definition 1.2 Let $H: X \times X \rightarrow Y$ be a mapping. $H$ is said to be affine in the second argument if, for each $X \in X$, $t_{i} \in R(i=1,2, \cdots, n)$ with $\quad \sum_{i=1}^{n} t_{i}=1$ such that for each $y_{i} \in X_{i}(i=1,2, \cdots, n) \quad$ we have $H\left(x, \sum_{i=1}^{n} t_{i} y_{i}\right)=\sum_{i=1}^{n} t_{i} H\left(x, y_{i}\right)$.

Similarly, we can define the affinity of $H$ in the first argument.

\section{EXISTENCE RESULTS}

Theorem 2.1 Let $X$ be a compact convex subset of a Banach space $E$ and $Y$ a Banach space with convex cone $P$ such that int $P \neq \phi$ and $P \neq Y$. Let $N: L(E, Y) \times L(E, Y) \times L(E, Y) \rightarrow L(E, Y) \quad$ be $\quad$ a continuous mapping. Suppose that:

(i) $M, S, T: X \rightarrow 2^{L(E, Y)}$ are lower semicontinuous;

(ii) $H: X \times X \rightarrow Y$ and $\eta: X \times X \rightarrow Y$ are continuous in the second argument, respectively, and $H(x, x)=\eta(x, x)=0$ for all $x \in X$; 
(iii) the multivalued mapping $w: X \rightarrow 2^{Y}$ defined by $W(x)=Y /\{-$ int $P\}$, has a closed graph in $X \times Y$;

(iv) for each $y \in X$,

$B_{y}=\{x \in X: \exists u \in M(y), v \in S(y), w \in T(y)$

such that $\langle N(u, v, w), \eta(x, y)\rangle+H(x, y)<0\}$

is convex. Then GMVVLI-1 is solvable.

Proof. Define a multivalued mapping $F: X \rightarrow 2^{X}$ by

$F(x)=\left\{\begin{array}{l}y \in X:\langle N(u, v, w), \eta(x, y)\rangle+H(x, y) \nless 0, \\ \forall u \in M(y), u \in S(y), w \in T(y)\end{array}\right\}$

$\forall x \in X$. We first prove that $F$ is a KKM mapping. Suppose to the contrary, $F$ is not a KKM mapping. Then the convex hull of every finite subset $\left\{x_{1}, x_{2}, \cdots, x_{n}\right\}$ of $X$ is not contained in the corresponding union $\bigcup_{i=1}^{n} F\left(x_{i}\right)$. Let $y \in \operatorname{co}\left\{x_{1}, x_{2}, \cdots, x_{n}\right\}$. Then $y=\sum_{i=1}^{n} \alpha_{i} x_{i}$ for some $\alpha_{i} \geq 0, i=1,2, \cdots, n \quad$ with $\quad \sum_{i=1}^{n} \alpha_{i}=1 \quad$ and $y \notin \bigcup_{i=1}^{n} F\left(x_{i}\right)$. Then we have for each $i \in\{1,2, \cdots, n\}, \exists u \in M(y), v \in S(y), w \in T(y)$, such that

$\left\langle N(u, v, w), \eta\left(x_{i}, y\right)\right\rangle+H\left(x_{i}, y\right)<0$.

Since by assumption (iv), $B_{y}$ is convex, then co $\left\{x_{1}, x_{2}, \cdots, x_{n}\right\} \subset B_{y}$,

$\left\langle N(u, v, w), \eta\left(\sum_{i=1}^{n} \alpha_{i} x_{i}\right)\right\rangle+H\left(\sum_{i=1}^{n} \alpha_{i} x_{i}, \sum_{i=1}^{n} \alpha_{i} x_{i}\right)$ $\in-$ int $P$

It follows from assumption (ii) that $0 \in-$ int $P$, which contradicts $P \neq Y$. Therefore $F$ is a KKM mapping. Next we prove that for any $x \in X, F(x)$ is closed. Indeed, Let $\left\{y_{n}\right\}$ be s sequence in $F(x)$ converging to $y^{*} \in X$. By the lower semicontinuity

of $M, S, T$, for $\forall\left(u^{*}, v^{*}, w^{*}\right) \in M\left(y^{*}\right) \times S\left(y^{*}\right) \times T\left(y^{*}\right)$, the re exist $u_{n} \in M\left(y_{n}\right), v_{n} \in S\left(y_{n}\right), w_{n} \in T\left(y_{n}\right)$ for all $n$ such that

$\left(u_{n}, v_{n}, w_{n}\right) \rightarrow\left(u^{*}, v^{*}, w^{*}\right) \in L(E, Y) \times L(E, Y) \times L(E, Y)$

Since $y_{n} \in F(x)$ for all $n$, we have

$$
\left\langle N\left(u_{n}, v_{n}, w_{n}\right), \eta\left(x_{n}, y_{n}\right)\right\rangle+H\left(x, y_{n}\right) \nless 0,
$$

Which implies that

$$
\left\langle N\left(u_{n}, v_{n}, w_{n}\right), \eta\left(x_{n}, y_{n}\right)\right\rangle+H\left(x, y_{n}\right) \in W\left(y_{n}\right) .
$$

It follows from assumption (ii) that $H$ and $\eta$ are continuous in the second argument, respectively, and note that $N$ is continuous, we has a closed graph in $X \times Y$ and $\left(u_{n}, v_{n}, w_{n}, y_{n}\right) \rightarrow\left(u^{*}, v^{*}, w^{*}, y^{*}\right)$, we get

$$
\begin{aligned}
& \left\langle N\left(u_{n}, v_{n}, w_{n}\right), \eta\left(x, y_{n}\right)+H\left(x, y_{n}\right)+H\left(x, y_{n}\right)\right\rangle \\
& \rightarrow\left\langle N\left(u^{*}, v^{*}, w^{*}\right), \eta\left(x, y^{*}\right)\right\rangle+H\left(x, y^{*}\right) \in W\left(y^{*}\right) .
\end{aligned}
$$

Meaning that $\left\langle N\left(u^{*}, v^{*}, w^{*}\right), \eta\left(x, y^{*}\right)\right\rangle+H\left(x, y^{*}\right) \nless 0$. Thus $y^{*} \in F(x)$ and $F(x)$ is closed. Furthermore, since $X$ is a compact subset of $E$ and $F(x) \subset X$ for each $x \in X$. Then $F(x)$ is compact. It follows from Lemma 1.1 that $\bigcap_{x \in X} F(x) \neq \phi$, that is, there exists $y \in X$ such that for any $(u, v, w) \in M(y) \times S(y) \times T(y)$, satisfying

$$
\langle N(u, v, w), \eta(x, y)\rangle+H(x, y) \nless 0, \forall x \in X,
$$

which implies that GMVVLI-1 is solvable. This completes the proof.

Corollary 2.1 Let $X$ be a compact convex subset of a Banach space $E$ and $Y$ a Banach space with convex cone $P$ such that, int $P \neq \phi$ and $P \neq Y$.

$N: L(E, Y) \times L(E, Y) \times L(E, Y) \rightarrow L(E, Y) \quad$ be $\quad$ a continuous mapping. Assume that

(i) $M, S, T: X \rightarrow 2^{L(E, Y)}$ are lower semicontinuous;

(ii) $H: X \times X \rightarrow Y, \eta: X \times X \rightarrow Y$ are continuous in the second argument and affine in the first argument, respectively, and $H(x, x)=\eta(x, x)=0$ for all $x \in X$;

(iii) the multivalued mapping $W: X \rightarrow 2^{Y}$ defined by $W(x)=Y /\{-$ int $P\}$, has a closed graph in $X \times Y$.

Then GMVVLI-1 is solvable.

Proof It is sufficient to prove that for each $y \in X$, the set

$$
B_{y}=\{x \in X: \exists u \in M(y), v \in S(y), w \in T(y)
$$

such that $\langle N(u, v, w), \eta(x, y)\rangle+H(x, y)<0\}$

is convex. For this, let $x_{1}, x_{2} \in B y$ and $\alpha, \beta \geq 0$ such that $\quad \alpha+\beta=1$. Then for some $u \in M(y), v \in S(y), w \in T(y)$, we have

$$
\left\langle N(u, v, w), \eta\left(x_{1}, y\right)\right\rangle+H\left(x_{1}, y\right) \in-\operatorname{int} P,
$$


and

$$
\left\langle N(u, v, w), \eta\left(x_{2}, y\right)\right\rangle+H\left(x_{2}, y\right) \in-\operatorname{int} P .
$$

Nothing that -int $\mathrm{P}$ is a convex cone and multiplying (1) by $\alpha$ and (2) by $\beta$ and adding, we have

$$
\begin{aligned}
& \alpha\left\langle N(u, v, w), \eta\left(x_{1}, y\right)\right\rangle+H\left(x_{1}, y\right)+\beta\left\langle N(u, v, w), \eta\left(x_{2}, y\right)\right\rangle+H\left(x_{2}, y\right) \\
& \in-(\alpha \operatorname{int} P+\beta \operatorname{int} P)=-\operatorname{int} P .
\end{aligned}
$$

It follows from the affinity of $H$ and $\eta$ in the first argument and (3) that

$$
\left\langle N(u, v, w), \alpha \eta\left(x_{1}, y\right)\right\rangle+\left\langle N(u, v, w), \beta \eta\left(x_{2}, y\right)\right\rangle
$$$$
+\alpha H(x, y)+\beta H\left(x_{2}, y\right)
$$

$=\left\langle N(u, v, w), \eta\left(\alpha x_{1}+\beta x_{2}, y\right)\right\rangle+H\left(\alpha x_{1}+\beta x_{2}, y\right) \in-\operatorname{int} P$.

That is, $\alpha x_{1}+\beta x_{2} \in B y$, thus $B_{y}$ is convex, completing the proof.
Remark 2.1 Theorem 2.1 and corollary 2.1 extend the corresponding results of [5].

Remark 2.2 If all the conditions of Theorem 2.1 and corollary 2.1 are satisfied, respectively, then GMVVLI-2 is solvable.

\section{References}

[1] Ansari, Q.H.: On generalized vector variational-like inequalities. Ann. Sci. Math. Quebec 19, 131-137(1995)

[2] Chen, G. Y.: Existence of solutions for a vector variational inequality: an extension of Hartman-Stampac-chia theorem. J. Optim. Theory Appl. 74, 445-456(1992)

[3] Kinderlehrer, D., Stampacchia, G. : An Introduction to Variational Inequalities and Their applications, in: pure and Applied Mathematics,vol.88, Academic Press, New York, 1980.

[4] Fan, K.: A generalization of Tychonoffs fixed point theorem. Math. Anal. 142, 305-310(1961)

[5] Irfan, S. S., Ahmad R.: Generalized multivalve vector variational-like inequalities .J.Glob.Optim.DOI 10. 1007/s 10898-009-9404-4. 2009, 16(2009). 